\title{
Tecnologías de la Información y la Comunicación en Salud: Análisis de Componentes Principales en la evaluación del desempeño competitivo ${ }^{1}$
}

\section{Information and Communication Technologies in Health: Principal Components Analysis in the evaluation of competitive performance}

\author{
J. F. Ramírez, V. G. López, A. R. Ramírez y M. Morejón
}

Recibido: marzo 11 de 2021 - Aceptado: noviembre 14 de 2021.

\begin{abstract}
Resumen- Las Tecnologías de la Información y la Comunicación constituyeron la clave en la irrupción de la Tercera Revolución Industrial en la década de 1950. Desde su surgimiento y hasta la fecha se han consolidado como un factor diferenciador en la administración estratégica y en la obtención de ventajas competitivas en la industria y el comercio. En el sector de la salud, las investigaciones sobre tecnologías y competitividad no demuestran igual auge, existiendo vacíos teóricos y prácticos en su análisis y medición. El objetivo de la investigación fue analizar el impacto que tienen las Tecnologías de la Información y la Comunicación en el desempeño competitivo de las instituciones de salud de Ensenada, Baja California, mediante el Análisis de Componentes Principales. La investigación es cuantitativa, con alcance correlacional y diseño experimental. La muestra empleada es representativa de la población objeto de análisis y la aplicación de cuestionarios posibilitó la obtención de los datos. Se aplicaron dos cuestionarios a profesionales de la salud $(n=203)$ y a pacientes $(n=271)$, se determinó la fiabilidad y la validez de constructo del instrumento de medición y se analizaron los datos obtenidos para arribar a conclusiones. Los resultados obtenidos demostraron que las
\end{abstract}

${ }^{1}$ Producto derivado del proyecto de investigación "Desarrollo de un modelo computacional de evaluación del desempeño competitivo de las instituciones prestadoras de servicios médicos, en el contexto de la Cuarta Revolución Industrial en Ensenada, Baja California", apoyado por la Universidad Autónoma de Baja California a través de la Maestría en Administración.

J. F. Ramírez, Universidad Autónoma de Baja California, Ensenada, México, email: jramirez14@uabc.edu.mx.

V. G. López, Universidad Autónoma de Baja California, Ensenada, México, email: virginia.lopez@uabc.edu.mx.

A. R. Ramírez, Universidad de Ciencias Médicas, Cienfuegos, Cuba, email: aliciar740205@gmail.com.

M. Morejón, Universidad Autónoma de Baja California, Ensenada, México, email: maylevis.morejon@uabc.edu.mx.

Como citar este artículo: Ramírez J. F., López, V. G., Ramírez, A. R. y Morejón, M. Tecnologías de la Información y la Comunicación en Salud: Análisis de Componentes Principales en la evaluación del desempeño competitivo, Entre Ciencia e Ingeniería, vol. 15, no. 30, pp. 22-29, juliodiciembre, 2021. DOI: https://doi.org/10.31908/19098367.2618.

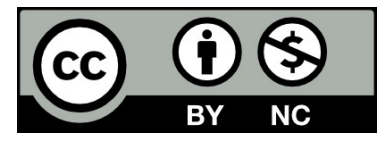

Attribution-NonCommercial 4.0 Intenational (CC By-NC 4.0)
Tecnologías de la Información y la Comunicación constituyen un factor de desempeño competitivo en salud. Además, impactan favorablemente en la mejora del proceso de atención médica, la calidad y los tiempos de atención a pacientes.

Palabras clave - administración estratégica, competitividad, salud, tecnologías de la información y la comunicación.

Abstract - Information and Communication Technologies were the key to the emergence of the Third Industrial Revolution in the 1950s. Since its inception and to date, they have been consolidated as a differentiating factor in strategic management and in obtaining competitive advantages in industry and commerce. In the health sector, research on technologies and competitiveness does not show the same growth, with theoretical and practical gaps in their analysis and measurement. The objective was to analyze the impact that Information and Communication Technologies have on the competitive performance of health institutions in Ensenada, Baja California, through the Principal Component Analysis. The research is quantitative, with correlational scope and experimental design. The sample used is representative of the population under analysis and the application of questionnaires made it possible to obtain the data. Two questionnaires were applied to health professionals $(n=203)$ and to patients $(n=271)$, the reliability and construct validity of the measurement instruments were determined, and the data obtained were analyzed to reach conclusions. The results obtained showed that Information and Communication Technologies constitute a competitive performance factor in health. In addition, they have a favorable impact on the improvement of the medical care process, the quality and the times of patient care.

Keywords - competitiveness, health, information and communication technologies, strategic management.

\section{INTRODUCCIÓN}

EGÚN diversos autores, para mantener un alto desempeño competitivo en la actualidad, las empresas deben posicionarse en las fronteras de la innovación [1], [2]. Para [3] y [4] la ejecución de una acertada estrategia de investigación e innovación debe incluir necesariamente los elementos en los que se basa la cuarta revolución que se está llevando a cabo en el ámbito industrial. Relacionado con la Cuarta Revolución Industrial, se ha demostrado en varios estudios como los realizados por [5]-[7], que la incorporación 
de las Tecnologías de la Información y la Comunicación (TIC), como área de conocimiento en la cual encausar las innovaciones e investigaciones, han constituido puntos clave en la elevación del desempeño competitivo de las empresas en diversos sectores económicos a nivel mundial.

En el sector de la salud, hoy en día también constituye una prioridad la elevación del desempeño competitivo de sus instituciones, por cada uno de sus niveles de atención [8]. Tal exigencia tiene el propósito de reducir los costos de atención y aumentar la productividad, específicamente en países altamente desarrollados y en vías de desarrollo [9], [10]. En estos países la demanda de servicios hospitalarios es cada vez mayor debido, entre otras cuestiones, al acelerado envejecimiento poblacional y al incremento de los costos asociados a la atención de enfermedades crónicas [11]-[13].

Las TIC dentro de la Cuarta Revolución Industrial han propiciado la explosión a escala mundial de descubrimientos científicos y tecnológicos disruptivos (por ejemplo: Big Data, Computación en la Nube, Internet de las Cosas, analíticas de datos, Machine Learning y Deep Learning, etc.), por medio de sistemas ciberfísicos, los cuales han establecido nuevos paradigmas en la forma de hacer las cosas, impactando de manera positiva y exponencial en los procesos de gestión y producción en la nueva sociedad del conocimiento [2]. Todos estos avances tienen en el sector de la salud un área de aplicación potencial presente y futura, para contribuir con la obtención de ventajas competitivas que propicien mejorar la calidad de vida de la población y la eficiencia en la gestión hospitalaria [14].

En la investigación realizada por [15], los autores concluyeron que el empleo de las TIC, desde su dimensión Atención al paciente, Gestión y Social, constituye un factor de desempeño competitivo en salud, desde su dimensión Control de recursos materiales, Tiempo de atención a pacientes, Calidad del proceso de atención, Eficiencia hospitalaria, Comportamiento organizacional y Relación interorganizacional. Diversos investigadores han abordado dicho fenómeno desde diferentes perspectivas. En el estudio realizado por [16], el autor evalúa el impacto de la aplicación de las TIC en un modelo de atención médica. Para ello, se hizo dos grupos de pacientes participantes, uno al cual se le realizó su proceso de atención de manera convencional y el otro mediante el uso de las TIC. Para el último grupo se constató una disminución en los tiempos de la consulta médica, los cuales mostraron niveles de satisfacción superior con el servicio ofrecido [17].

Según [16], [18] y [19] el empleo de las TIC en el proceso de atención a pacientes es beneficioso. Favorece la disminución de errores en la prescripción médica y adherencia a las prácticas clínicas, lo que mejora los procesos de atención médica, disminuyendo los tiempos de atención. Asimismo, en las instituciones hospitalarias los administradores tienen intereses elevados de aplicar las TIC en función de mejorar la eficiencia y efectividad hospitalaria [20]. Se ha constatado que con su empleo en los hospitales se evidencia un desempeño superior, tanto en el control de los recursos, como en su utilización efectiva [21]. Finalmente, las TIC constituye una herramienta estratégica para el desarrollo organizacional, ya que eleva los estándares de calidad, mediante la inclusión social. Tienen un impacto positivo en el bienestar de los pacientes, al favorecer la comunicación en el proceso asistencial [22]-[24].

Por su parte, en el estudio llevado a cabo por [25], los resultados obtenidos les permitieron confirmar la importancia que tienen las TIC en los eslabones críticos, como parte del proceso de atención médica, tanto en la disminución de los tiempos, no ocurrencia de cuellos de botella en los servicios, calidad de la atención prestada, así como en la disminución de errores por medicación inapropiada o codificación incorrecta. En este contexto, los trabajos de [19] y [24] confirman la dimensión social de las TIC, siendo herramientas estratégicas para el desarrollo organizacional, cuya adopción eleva los estándares de calidad, mediante la inclusión social de pacientes de culturas distintas. Tal afirmación es soportada por [22], quienes consideran que su empleo ha sido y puede ser utilizado eficientemente para resolver problemáticas asociadas con el paciente y su entorno. Igualmente, según refieren [23] y [26], en sus estudios evalúan cómo las TIC son herramientas que facilitan la comunicación durante todo el proceso de atención médica.

En un estudio empírico realizado por [27], llevado a cabo en 382 hospitales en Taiwán, se identificaron dos formas esenciales de cooperación, las redes de salud hospitalarias y los sistemas de salud. Se evidenció que, si bien ambos tienen un efecto positivo en el desempeño organizacional, los sistemas de salud tienen mayores efectos sobre la eficiencia hospitalaria, constituyendo las TIC un detonante en las relaciones favorables entre sistemas de salud en los últimos años [28] - [30].

Teniendo en cuenta los antecedentes presentados, el objetivo de la investigación es analizar el impacto que tienen las Tecnologías de la Información y la Comunicación en el desempeño competitivo de las instituciones de salud de Ensenada, Baja California, mediante el Análisis de Componentes Principales.

\section{METODOLOGÍA}

La investigación es cuantitativa, con alcance correlacional. La hipótesis de la investigación consiste en demostrar que las TIC impactan en el desempeño competitivo de las instituciones de salud en Ensenada, Baja California, México. Para ello, la investigación estuvo compuesta por tres fases:

1. Aplicación de cuestionarios a profesionales de la salud y a pacientes

2. Análisis de fiabilidad $y$ validez de constructo del instrumento de medición (cuestionarios)

3. Análisis de tipo comparativo y correlacional, a partir de los datos obtenidos

Según [31], el cuestionario es la técnica de obtención de información más empleada en las ciencias sociales. Es por ello que fueron aplicados dos cuestionarios, a profesionales de la salud y a pacientes. De esta manera se puede analizar desde diferentes perspectivas el impacto real que tienen las TIC en el desempeño competitivo de las instituciones de salud en Ensenada, Baja California, lo que propicia un mejor análisis, triangulación de información y obtención de conclusiones más fiables. Los profesionales de la salud encuestados se 
comprenden en cuatro categorías: Administrativo, Enfermero/Médico (General o de Especialidad), Odontólogo y Trabajador de Laboratorio. Los resultados obtenidos fueron analizados con el paquete estadístico IBM SPSS Statistics.

Las poblaciones objeto de estudio son infinitas, debido a que no se conoce con exactitud la cantidad de profesionales de la salud que laboran en las instituciones de salud en Ensenada, Baja California, como tampoco el total de pacientes que se atienden en las mismas. Es por ello que el cálculo de las muestras se emplea la siguiente fórmula, propuesta por [32]:

$$
n=\frac{Z^{2} p(1-p)}{e^{2}}
$$

En donde, para el caso de los profesionales de la salud:

$\mathrm{n}=$ es el tamaño de la muestra de profesionales de la salud a obtener.

$\mathrm{Z}=$ es el valor obtenido mediante niveles de confianza, $90 \%$ (1.645), siendo confiable.

$\mathrm{p}=$ proporción de elementos que responden satisfactoriamente, siendo 0.25

$\mathrm{e}=$ representa el límite aceptable de error muestral, siendo 5\% (0.05) el empleado.

Para el caso de los pacientes:

$\mathrm{n}=$ es el tamaño de la muestra de pacientes a obtener.

$\mathrm{Z}=$ es el valor obtenido mediante niveles de confianza, $90 \%$ (1.645), siendo confiable.

$\mathrm{p}=$ proporción de elementos que responden satisfactoriamente, siendo 0.5

$\mathrm{e}=$ representa el límite aceptable de error muestral, siendo 5\% (0.05) el empleado.

Como resultado del cálculo anterior, el tamaño de la muestra de profesionales de la salud encuestados es 203 y el de pacientes es 271, para un total de 474 cuestionarios aplicados. De esta manera se contribuye con que la muestra sea representativa de la población, siendo generalizables a todos sus miembros los resultados obtenidos en el estudio.

\section{III.RESULTADOS}

\section{A. Aplicación de cuestionarios a profesionales de la salud y a pacientes}

En la aplicación de los cuestionarios a profesionales de la salud y pacientes, se llevó a cabo un plan de acción determinado por:

1. El procedimiento: comprendió las acciones realizadas, las condiciones que se tuvieron en cuenta y la vía mediante la cual se estableció el contacto con los participantes.

2. El diseño del instrumento: abordó la estructura del instrumento de medición diseñado, la cantidad de preguntas y escalas de medida empleadas, así como su origen. El índice de validez de contenidos obtenido, producto del jueceo de expertos aplicado al instrumento, arrojó un valor de 0.79 , siendo adecuado.

3. El escenario de aplicación: describió la vía de administración del instrumento de medición a los encuestados, así como los aspectos relevantes tenidos en cuenta en su aplicación, para que no afectaran en mayor medida en la calidad de la información obtenida.

4. Los participantes objetivos: indicó el número de encuestados por instrumento de medición y las principales características de los mismos. Además, incluyó los criterios de inclusión o exclusión que fueron incorporados para una adecuada aplicación de la investigación.

En la aplicación de los cuestionarios el único criterio restrictivo fue la no inclusión en el estudio de profesionales de la salud y pacientes que no pertenecieran a Ensenada, Baja California, debido a que constituyeron la muestra seleccionada. En el caso de los pacientes no hubo criterios adicionales de restricción, debido a que todos de una u otra manera son beneficiaros de los servicios de salud, siendo sus opiniones valiosas para la investigación.

Se tuvo en consideración la situación epidemiológica existente, producto del COVID-19 y las restricciones que imposibilitan el contacto físico en las instituciones de salud. Es por ello que el cuestionario fue automatizado, utilizando el software de administración de encuestas Google Forms y distribuido entre las personas mediante las redes sociales (Facebook, Twitter, Telegram e Instagram) y el correo electrónico. El sesgo en la obtención de información que puede producirse en dicha aplicación fue otro de los aspectos que influyeron en la aplicación de cuestionarios a profesionales de la salud y a pacientes, de manera que la triangulación entre ambos datos obtenidos contrarrestara la existencia de sesgo y aumentara la calidad y confiabilidad de la información obtenida para posteriores análisis, resultados y conclusiones.

El cuestionario aplicado a profesionales de la salud tiene 59 preguntas, el cual fue desarrollado en su totalidad en la investigación. Las 59 preguntas son cerradas, las cuales responden a las nueve dimensiones analizadas en la investigación:

- Tres dimensiones de la variable independiente TIC (Atención al paciente, Gestión y Social)

- Seis dimensiones de la variable dependiente Desempeño Competitivo

(Control de recursos materiales, Tiempo de atención a pacientes, Calidad del proceso de atención, Eficiencia hospitalaria, Comportamiento organizacional, Relación inter-organizacional).

Cuatro preguntas son medidas en escala nominal, mientras que 55 preguntas son evaluadas en escala ordinal. En cuanto al tipo de respuesta, de acuerdo con la naturaleza de las variables cualitativas, tres son dicotómicas, mientras que 56 son politómicas o de selección múltiple.

Las respuestas dicotómicas responden a la escala nominal con respuestas de Sí-No. En cambio, las respuestas politómicas hacen alusión a dos tipos de escala ordinal con respuestas de (Totalmente en desacuerdo, En desacuerdo, De acuerdo, Totalmente de acuerdo) y (Nunca, Casi nunca, casi siempre, Siempre). 
El cuestionario aplicado a pacientes solo incorpora los reactivos del cuestionario aplicado a profesionales de la salud para las dimensiones Social, Tiempo de atención a pacientes y Calidad del proceso de atención, en las cuales los pacientes también tienen implicación. De esta manera, el cuestionario aplicado a pacientes tiene 19 preguntas.

TABLA I

ANÁLISIS DE FIABILIDAD Y ANÁLISIS FACTORIAL EXPLORATORIO MEDIANTE EL ANÁLISIS DE COMPONENTES PRINCIPALES. CUESTIONARIO A PROFESIONALES DE LA SALUD. FUENTE: ELABORACIÓN PROPIA CON DATOS OBTENIDOS DEL SPSS.

\begin{tabular}{|c|c|c|c|c|c|c|}
\hline \multirow{2}{*}{ Componente } & \multicolumn{3}{|c|}{ Autovalores iniciales } & \multicolumn{3}{|c|}{$\begin{array}{l}\text { Sumas de extracción de cargas al } \\
\text { cuadrado }\end{array}$} \\
\hline & Total & $\begin{array}{c}\% \mathrm{de} \\
\text { varianza }\end{array}$ & $\begin{array}{c}\% \\
\text { acumulado }\end{array}$ & Total & $\begin{array}{l}\% \text { de } \\
\text { varianza }\end{array}$ & $\begin{array}{c}\% \\
\text { acumulado }\end{array}$ \\
\hline 1 & 9,341 & 23,353 & 23,353 & 9,341 & 23,353 & 23,353 \\
\hline 2 & 7,152 & 17,881 & 41,234 & 7,152 & 17,881 & 41,234 \\
\hline 3 & 5,948 & 14,870 & 56,104 & 5,948 & 14,870 & 56,104 \\
\hline 4 & 4,016 & 10,040 & 66,144 & 4,016 & 10,040 & 66,144 \\
\hline 5 & 2,689 & 6,722 & 72,866 & 2,689 & 6,722 & 72,866 \\
\hline 6 & 1,500 & 3,750 & 76,616 & 1,500 & 3,750 & 76,616 \\
\hline 7 & 1,266 & 3,165 & 79,782 & 1,266 & 3,165 & 79,782 \\
\hline 8 & 1,250 & 3,124 & 82,906 & 1,250 & 3,124 & 82,906 \\
\hline 9 & 1,023 & 2,559 & 85,465 & 1,023 & 2,559 & 85,465 \\
\hline 10 &, 866 & 2,164 & 87,629 & & & \\
\hline 11 & 689 & 1,722 & 89,350 & & & \\
\hline 12 & ,644 & 1,610 & 90,960 & & & \\
\hline 13 &, 576 & 1,439 & 92,399 & & & \\
\hline 14 & ,509 & 1,273 & 93,672 & & & \\
\hline 15 & ,446 & 1,114 & 94,786 & & & \\
\hline 59 & ,006 &, 014 & 100,000 & & & \\
\hline \multicolumn{6}{|c|}{ Coeficiente Alfa de Cronbach total del instrumento (59 ítems) } & 816 \\
\hline \multicolumn{7}{|c|}{ Prueba de KMO y Bartlett } \\
\hline \multicolumn{6}{|c|}{ Medida Kaiser-Meyer-Olkin de adecuación de muestreo } &, 852 \\
\hline \multirow[t]{3}{*}{ Prueba d } & esfericic & d de Bart & \multicolumn{3}{|c|}{ Aprox. Chi-cuadrado } & 1364,836 \\
\hline & \multicolumn{5}{|c|}{$\mathrm{gl}$} & 406 \\
\hline & \multicolumn{5}{|c|}{ Sig. } & 000 \\
\hline
\end{tabular}

\section{B. Análisis de fiabilidad y validez de constructo}

Luego de la aplicación de los cuestionarios, se evaluó la confiabilidad de ambos instrumentos de medición a través del coeficiente Alfa de Cronbach. Asimismo, se aplicó la técnica estadística Análisis Factorial Exploratorio (AFE), por medio del Análisis de Componentes Principales (PCA), para evaluar la validez de constructo. Los resultados se muestran en las tablas I y II.

Los valores de confiabilidad son superiores a 0.7 e inferiores a 0.9 en ambos instrumentos de medición, lo cual evidencia su adecuada confiabilidad, no existiendo redundancia. Además, en el AFE se puede concluir que:

- En la medida Kaiser-Meyer-Olkin (KMO) de adecuación del muestreo, la cual evalua el grado de correlación entre variables, se obtuvo valores superiores a 0.70 , siendo muy bueno, pudiendo aplicarse el AFE.

- En la prueba de esfericidad de Bartlett, en la cual se determina si las correlaciones entre variables son significativas, se obtuvo resultados significativos para valores de esfericidad de Bartlett de 1364.836 (profesionales de salud) y 1029.656 (pacientes), para 406 y 171 grados de libertad respectivamente. Todo lo anterior hace indicar que a ambos cuestionarios se les puede aplicar la técnica de AFE de manera apropiada.

- En el método PCA, los resultados evidencian la existencia de nueve factores (cuestionario aplicado a profesionales de la salud) y tres factores (cuestionario aplicado a pacientes), que se corresponden con la misma cantidad de dimensiones evaluadas en cada uno de los cuestionarios. En cada caso, todos los factores tienen autovalores superiores a 1, necesario para aplicar análisis exploratorios. Los factores extraídos explican el $85.46 \%$ y el $77.71 \%$ de la varianza total, siendo muy buenos. Adicionalmente, todos los factores presentan cargas factoriales superiores a 0.50 siendo favorable también.

TABLA II

ANÁLISIS DE FIABILIDAD Y ANÁLISIS FACTORIAL EXPLORATORIO MEDIANTE EL ANÁLISIS DE COMPONENTES PRINCIPALES. CUESTIONARIO A PACIENTES. FUENTE: ELABORACIÓN PROPIA CON DATOS OBTENIDOS DEL SPSS. Varianza total explicada

\begin{tabular}{|l|r|r|r|r|r|r|}
\hline \multirow{2}{*}{ Componente } & \multicolumn{7}{|c|}{ Autovalores iniciales } & \multicolumn{3}{c|}{ Sumas de extracción de cargas al } \\
cuadrado
\end{tabular}

Método de extracción: análisis de componentes principales.

\section{Análisis de tipo comparativo y correlacional, a partir de los datos obtenidos}

Posteriormente, se realizó un análisis comparativo con los resultados obtenidos, donde se evaluó si el grado de penetración de las TIC existente en las instituciones de salud 
impacta estadísticamente en el desempeño competitivo de las mismas, evaluado en una mejor atención al paciente en consulta, en una mayor calidad del proceso de atención y en la consecución de tiempos de atención adecuados.

Tales resultados también fueron evaluados por tipos de institución de salud, privada o pública. Las variables objeto de análisis, su descripción y la escala con que fue medida se muestra en la Tabla III.

\section{TABLA III}

DESCRIPCIÓN DE LAS VARIABLES CONSIDERADAS PARA LA COMPARACIÓN

\begin{tabular}{|c|c|c|}
\hline Variable & Descripción & Escala de medición \\
\hline $\begin{array}{l}\text { Penetración } \\
\text { de las TIC }\end{array}$ & $\begin{array}{l}\text { Grado de penetración de las } \\
\text { TIC en la institución de salud, } \\
\text { evaluado a partir de su uso en } \\
\text { la ejecución de los procesos } \\
\text { asistenciales, de promoción y } \\
\text { prevención de salud, así como } \\
\text { de apoyo al diagnóstico médico }\end{array}$ & $\begin{array}{l}\text { Nominal: } \\
\text { 1. Bajo } \\
\text { 2. Medio } \\
\text { 3. Alto }\end{array}$ \\
\hline $\begin{array}{l}\text { Atención al } \\
\text { paciente en } \\
\text { consulta }\end{array}$ & $\begin{array}{l}\text { Engloba el impacto de las TIC } \\
\text { por los médicos para brindar } \\
\text { una mejor atención sanitaria, } \\
\text { desde su evaluación en la } \\
\text { asistencia médica y para la } \\
\text { promoción de salud, } \\
\text { prevención, diagnóstico y } \\
\text { tratamiento de enfermedades }\end{array}$ & $\begin{array}{l}\text { Ordinal: } \\
\text { 0. Totalmente en } \\
\quad \text { desacuerdo } \\
\text { 1. En desacuerdo } \\
\text { 2. De acuerdo } \\
\text { 3. Totalmente de } \\
\quad \text { acuerdo }\end{array}$ \\
\hline $\begin{array}{l}\text { Tiempo de } \\
\text { atención }\end{array}$ & $\begin{array}{l}\text { Aborda la contribución e } \\
\text { impacto de las TIC en la } \\
\text { consecución de tiempos de } \\
\text { atención médica más óptimos } \\
\text { - Estudios indican que un } \\
\text { tiempo éticamente aceptable } \\
\text { de consulta médica debe } \\
\text { oscilar entre } 16 \text { y } 20 \text { min } \\
\text { [33]. }\end{array}$ & $\begin{array}{l}\text { Ordinal: } \\
\text { 1. Menos de } 10 \text { minutos } \\
\text { 2. Más de } 30 \text { minutos } \\
\text { 3. Entre } 10 \text { y } 15 \text { minutos } \\
\text { 4. Entre } 16 \text { y } 20 \\
\text { minutos } \\
\text { 5. Entre } 20 \text { y } 30 \\
\text { minutos }\end{array}$ \\
\hline $\begin{array}{l}\text { Calidad del } \\
\text { proceso de } \\
\text { atención }\end{array}$ & $\begin{array}{l}\text { Engloba el impacto de las TIC } \\
\text { desde su contribución al } \\
\text { bienestar de la población, } \\
\text { desde su evaluación en la } \\
\text { calidad de vida del paciente, la } \\
\text { equidad y accesibilidad a los } \\
\text { servicios de salud y la E-salud }\end{array}$ & $\begin{array}{l}\text { Ordinal: } \\
\text { 0. Totalmente en } \\
\quad \text { desacuerdo } \\
\text { 1. En desacuerdo } \\
\text { 2. De acuerdo } \\
\text { 3. Totalmente de } \\
\quad \text { acuerdo }\end{array}$ \\
\hline
\end{tabular}

En las Tabla IV se resume el análisis comparativo realizado, en donde la hipótesis nula es: La distribución de Penetración de las TIC es la misma entre las categorías de Identificador hospitalario. Luego de evaluados los resultados, el estadístico de contraste chi-cuadrado de Pearson devuelve un valor de 40,402. Asimismo, se obtiene un $p \_v a l o r=0.000$, que al ser menor que 0,05 demuestra que existen diferencias significativas entre las muestras comparadas, por lo que se rechaza la hipótesis nula.

Además, como se muestra en la tabulación cruzada la diferencia significativa es a favor de los hospitales privados, donde se reconoce una asociación significativa entre las variables categóricas "Hospital privado" y una elevada "Penetración de las TIC".

En la Tabla V se resume el análisis comparativo realizado, en donde la hipótesis nula es: Las distribuciones de Atención al paciente en consulta, Calidad del proceso de atención y Tiempo de atención son los mismos entre las categorías de
Penetración de las TIC. Luego de evaluados los resultados, se obtiene un p_valor $=0.000$ en todos los casos, que al ser menor que 0,05 demuestra que existen diferencias significativas entre las muestras comparadas. Adicionalmente, las significaciones asintóticas obtenidas del contraste no paramétrico $\mathrm{H}$ de Kruskal-Wallis hace indicar que se acepta la hipótesis de investigación, rechazándose la hipótesis nula. Adicionalmente, las diferencias significativas pueden ser comprobadas en el histograma de la Fig. 1, donde se evidencian valores superiores de percepción de satisfacción en la variable Atención al paciente en consulta, en la medida que aumenta la penetración de las TIC en las instituciones de salud.

TABLA IV

PRUEBA DE CHI-CUADRADO Y TABULACIÓN CRUZADA (IDENTIFICADOR HOSPITALARIO Y PENETRACIÓN DE LAS TIC). FUENTE: ELABORACIÓN PROPIA CON DATOS OBTENIDOS DEL SPSS.

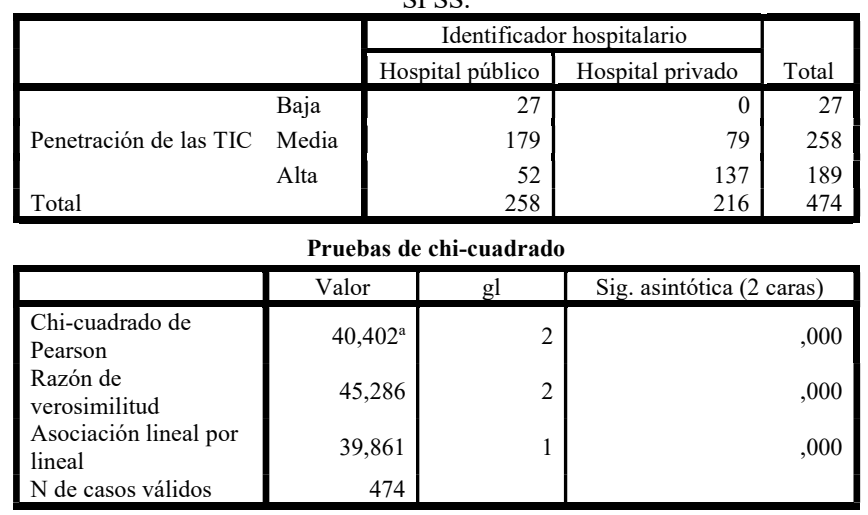

a. 0 casillas $(0,0 \%)$ han esperado un recuento menor que 5 . El recuento mínimo esperado es 5,01 .

TABLA V

RECUENTO Y CONTRASTE NO PARAMÉTRICO H DE KRUSKALWALLIS. FUENTE: ELABORACIÓN PROPIA CON DATOS OBTENIDOS DEL SPSS.

\begin{tabular}{|ll|r|r|r|r|}
\hline & & \multicolumn{2}{|c|}{ Atención al paciente en consulta } & \multirow{2}{*}{} \\
\cline { 3 - 6 } & & $\begin{array}{c}\text { En } \\
\text { desacuerdo }\end{array}$ & $\begin{array}{c}\text { De } \\
\text { acuerdo }\end{array}$ & $\begin{array}{c}\text { Totalmente } \\
\text { de acuerdo }\end{array}$ & \multirow{2}{*}{ Total } \\
\hline Penetración de & Baja & 27 & 0 & 0 & 27 \\
las TIC & Media & 0 & 238 & 20 & 258 \\
& Alta & 0 & 0 & 189 & 189 \\
Total & & 27 & 238 & 209 & 474 \\
\hline
\end{tabular}

Estadísticos de pruebaa ${ }^{a, b}$

\begin{tabular}{|l|r|}
\hline & $\begin{array}{c}\text { Atención al paciente } \\
\text { en consulta }\end{array}$ \\
\hline Chi-cuadrado & 165,155 \\
gl & 2 \\
Sig. asintótica &, 000 \\
\hline
\end{tabular}

a. Prueba de Kruskal Wallis

b. Variable de agrupación:

Penetración de las TIC

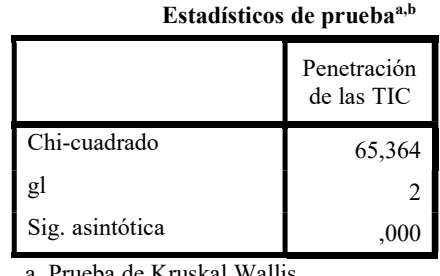

a. Prueba de Kruskal Wallis

b. Variable de agrupación: Tiempo de atención

Estadísticos de prueba ${ }^{a, b}$

\begin{tabular}{|c|c|}
\hline & Penetración de las TIC \\
\hline $\begin{array}{l}\text { Chi-cuadrado } \\
\text { gl } \\
\text { Sig. asintótica }\end{array}$ & $\begin{array}{r}65,390 \\
2 \\
, 000\end{array}$ \\
\hline
\end{tabular}




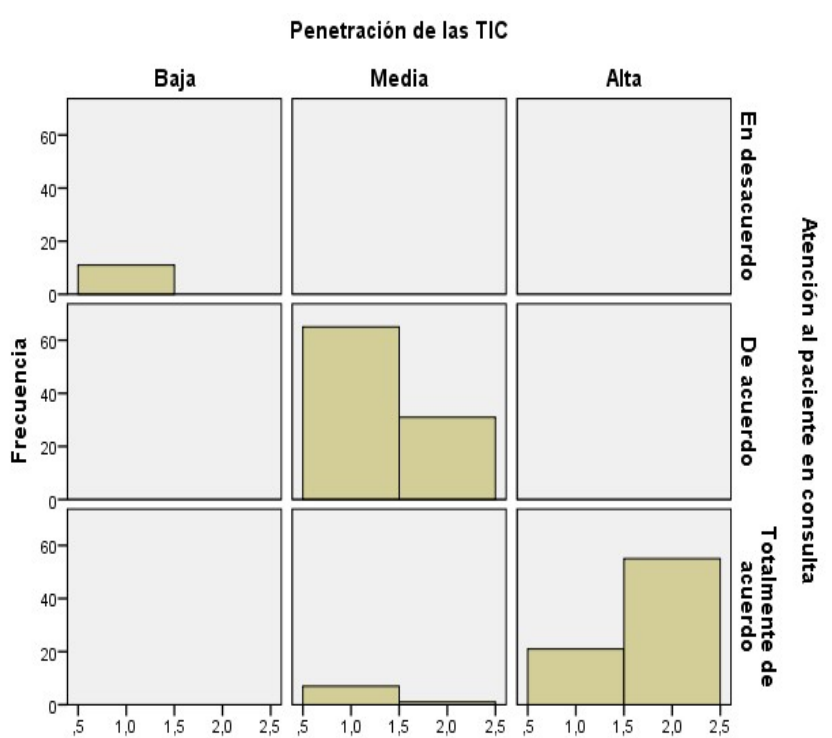

Fig. 1. Histograma con el análisis del comportamiento de las variables Penetración de las TIC y Atención al paciente en consulta. Fuente: elaboración propia con datos obtenidos del SPSS.

TABLA VI

ANÁLISIS DE CORRELACIÓN POR MEDIO DEL COEFICIENTE DE SPEARMAN. FUENTE: ELABORACIÓN PROPIA CON DATOS OBTENIDOS DEL SPSS.

\begin{tabular}{|c|c|c|c|c|}
\hline & & & $\begin{array}{l}\text { Penetración de } \\
\text { las TIC }\end{array}$ & $\begin{array}{l}\text { Calidad del } \\
\text { proceso de } \\
\text { atención }\end{array}$ \\
\hline \multirow{6}{*}{$\begin{array}{l}\text { Rho de } \\
\text { Spearman }\end{array}$} & \multirow{3}{*}{$\begin{array}{l}\text { Penetración } \\
\text { de las TIC }\end{array}$} & $\begin{array}{l}\text { Coeficiente de } \\
\text { correlación }\end{array}$ & 1,000 &, $826^{* *}$ \\
\hline & & Sig. (bilateral) & . & , 000 \\
\hline & & $\mathrm{N}$ & 474 & 474 \\
\hline & \multirow{3}{*}{$\begin{array}{l}\text { Calidad del } \\
\text { proceso de } \\
\text { atención }\end{array}$} & $\begin{array}{l}\text { Coeficiente de } \\
\text { correlación }\end{array}$ &, $826^{* *}$ & 1,000 \\
\hline & & Sig. (bilateral) & , 000 & . \\
\hline & & $\mathrm{N}$ & 474 & 474 \\
\hline
\end{tabular}

Teniendo en cuenta los resultados obtenidos, a continuación se analiza si la penetración percibida de las Tecnologías de la Información y la Comunicación (TIC) en las instituciones de salud de Ensenada, Baja California, guarda alguna relación con la calidad del proceso de atención médica que reciben los pacientes en las mismas. Como las variables a analizar están medidas en escala ordinal, se procede a utilizar el coeficiente de correlación de Spearman para efectuar los análisis.

Para ello, en la Tabla VI se calcula el coeficiente de correlación de Spearman, arrojando un $\mathrm{p}_{\text {_valor }}=0.826$. Este valor indica que existe una correlación alta, por lo que la relación existente entre ambas variables es una relación marcada. Adicionalmente, como se muestra en la Fig. 2, se está en presencia de una relación lineal positiva, debido a que en la medida que aumenta la penetración de las TIC en las instituciones de salud, se observa un aumento en la calidad de la atención al paciente, no indicando esto en ningún caso la existencia de causalidad.

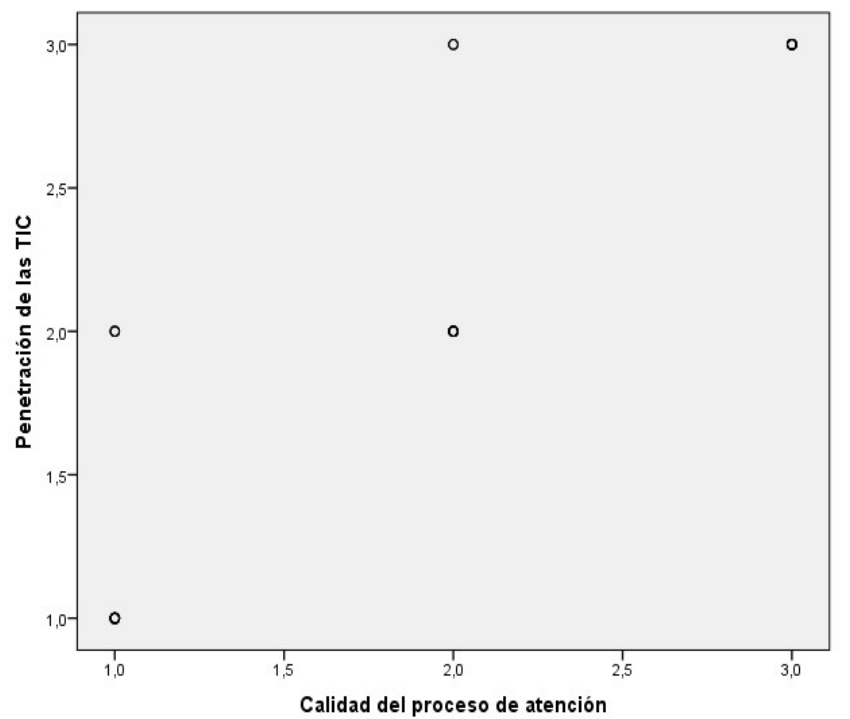

Fig. 2. Gráfico de dispersión en el análisis de relación de las variables Penetración de las TIC y Calidad del proceso de atención. Fuente: elaboración propia con datos obtenidos del SPSS.

\section{CONCLUSIONES}

En la investigación se analizó y constató el impacto que tienen las TIC en el desempeño competitivo de las instituciones de salud de Ensenada, Baja California. Para ello, se aplicaron cuestionarios a profesionales de la salud y a pacientes. Posteriormente, se llevó a cabo un análisis de fiabilidad y de validez de constructo, obteniéndose resultados positivos. Por último, se realizaron diversos análisis de tipo comparativo y correlacional, a partir de los datos obtenidos, que posibilitaron demostrar la hipótesis planteada en la investigación.

En el estudio se evidenció que, si bien las TIC constituyen un factor de desempeño competitivo en el sector de la salud, se observó un mayor empleo de tecnología en los hospitales privados, en comparación con los hospitales públicos. La misma se evaluó como una correlación alta, por tanto, relación marcada, caracterizada por ser una relación lineal positiva.

De igual manera, se percibe por parte de profesionales de la salud y de pacientes que las TIC impactan en mayor medida en una mejoría de los tiempos de atención y los tiempos de espera en las instituciones de salud, así como en el proceso de atención al paciente en consulta y en la calidad de la atención, estos últimos evaluados desde la promoción de salud, prevención, diagnóstico y tratamiento de enfermedades, así como en la disponibilidad, equidad y accesibilidad a los servicios de salud.

Los resultados obtenidos, si bien no pueden ser generalizables a toda la población, constatan que el empleo de las TIC puede constituir un factor de desempeño competitivo en las instituciones de salud en México, siendo la investigación un referente actualizado en el estudio del tema. Asimismo, las TIC se pueden considerar como un factor de administración estratégica.

Como limitación de la investigación estuvieron las condiciones en que se recopiló la información y su aplicabilidad solo a las instituciones de salud en Ensenada, Baja California. Como trabajos futuros en esta área de 
conocimiento y aplicación se recomienda el análisis de los efectos que provoca el empleo de las TIC para elevar el desempeño competitivo de las instituciones de salud en sus áreas asistenciales y administrativas, así como su contribución como un factor de administración estratégica en el contexto de la Cuarta Revolución Industrial.

\section{REFERENCIAS}

[1] N. Muljani and L. Ellitan, "Developing Competitiveness in Industrial Revolution 4.0," International Journal of Trend in Research and Development, vol. 6, no. 1, pp. 1-3, 2019. [En línea]. Disponible en http://repository.wima.ac.id/20321/

[2] K. Schwab, The fourth industrial revolution. First Edition. New York, USA: Crown Business, 2017.

[3] L. A. Bearzotti, "Industria 4.0 y la Gestión de la Cadena de Suministro: el desafío de la nueva revolución industrial," Gaceta Sansana, vol. 3, no. 8, 2018. [En línea]. Disponible en http://publicaciones.usm.edu.ec/index.php/GS/article/view/103.

[4] A. A. Sánchez, "La Industria 4.0. Análisis y estudio desde el Derecho en la 4ta Revolución Industrial," Advocatus, vol. 32, pp. 133-164, 2019. [En línea]. Disponible en

https://dialnet.unirioja.es/servlet/articulo?codigo $=7380434$.

[5] M. D. Singh and V. Ahila, "Organizational Climate and Information Technology on the Competitiveness of ICT Companies," Our Heritage, vol. 68 , no. 30, pp. 7072-7076, 2020. [En línea]. Disponible en https://doi.org/10.32628/IJSRSET207332.

[6] D. Skunca, M. Bogavac and D. Rudic, "Competitiveness of Small and Medium Enterprises in the Brics Countries and Serbia," Edited by Zoran Čekerevac. MEST Journal, pp. 92-98, 2019. [En línea]. Disponible en https://mest.meste.org/MEST_Najava/XIII_Skunca.pdf.

[7] K. Q. Thai and M. Noguchi, "Evaluating efficiency of English acute foundation trusts under system reform: a two-stage DEA approach," Health Services and Outcomes Research Methodology, vol. 19, no. 4, pp. 215-240, 2019. [En línea]. Disponible en https://link.springer.com/article/10.1007/s10742-019-00203-6.

[8] J. F. R. Pérez, T. R. Rodríguez, D. O. Fajardo y M. M. Valdes, "Componente para la toma de decisiones en salud. Un enfoque de análisis de redes sociales desde la minería de procesos," Revista cubana de informática médica, vol. 8, no. 1, pp. 46-63, 2016. [En línea]. Disponible en http://scielo.sld.cu/scielo.php?pid=S1684$18592016000100004 \&$ script $=$ sci arttext\&tlng=en.

[9] H. G. Calderón, J. E. L. Rúa y J. S. García, "Innovación en instituciones de salud como fuente de competitividad," Revista Venezolana de Gerencia, vol. 21, no. 74, pp. 227-241, 2016. [En línea]. Disponible en https://www.redalyc.org/pdf/290/29046685004.pdf.

[10] T. Mayer and K. Jensen, "Hardwiring hospital-wide flow to drive sustainable competitive performance," Management in Healthcare, vol. 2, no. 4, pp. 373-387, 2018. [En línea]. Disponible en https://www.ingentaconnect.com/content/hsp/mih/2018/00000002/0000 0004/art00009.

[11] J. Kalseth and T. Halvorsen, "Health and care service utilisation and cost over the life-span: a descriptive analysis of population data," BMC health services research, vol. 20, pp. 1-14, 2020. [En línea]. Disponible en https://doi.org/10.1186/s12913-020-05295-2.

[12] S. Ogura and M. M. Jakovljevic, "Global Population Aging-Health Care, Social and Economic Consequences," Frontiers in public health, vol. 6, pp. 335, 2018. [En línea]. Disponible en https://doi.org/10.3389/fpubh.2018.00335.

[13] R. Ofori-Asenso, K. L. Chin, A. J. Curtis, E. Zomer, S. Zoungas and D. Liew, "Recent patterns of multimorbidity among older adults in highincome countries," Population health management, vol. 22, no. 2, pp. 127-137, 2019. [En línea]. Disponible en https://doi.org/10.1089/pop.2018.0069.

[14] J. M. Valencia-Moreno, E. G. López, J. F. R. Pérez, J. P. F. Rodríguez and O. Á. Xochihua, "Exploring Breast Cancer Prediction for Cuban Women," In International Conference on Information Technology \& Systems, pp. 480-489, 2020. Springer, Cham. [En línea]. Disponible en https://link.springer.com/chapter/10.1007/978-3-030-40690-5_47

[15] J. F. R. Pérez, V. G. López-Torres and M. Morejón-Valdés, "Information and Communication Technologies as a competitive performance factor in provider institutions of medical services in Ensenada, Baja California," Journal of Administrative Science, vol. 2, no. 4, pp. 31-37, 2021. [En línea]. Disponible en https://doi.org/10.29057/jas.v2i4.6750.

[16] P. Robledo-Madrid, "Impacto de la aplicación de las Tecnologías de la Información (TIC) en un modelo de atención médica," Revista de Sanidad Militar, vol. 73, no. 2, pp. 96-104, 2020. [En línea]. Disponible en https://revistasanidadmilitar.org/index.php/rsm/article/view/17.

[17] J. A. Almaguer, R. H. García and V. V. Vargas, "Metodología intercultural para la realización de consultas informadas," Subsecretaría de Integración y Desarrollo del Sector Salud, Secretaría de Salud, 2013.

[18] M. Mukred, Z. M. Yusof, U. A. Mokhtar, A. S. Sadiq, B. Hawash and W. A. Ahmed, "Improving the decision-making process in the higher learning institutions via electronic records management system adoption," KSII Transactions on Internet and Information Systems, vol. 15, no. 1, pp. 90-113, 2021. [En línea]. Disponible en https://wlv.openrepository.com/handle/2436/623909.

[19] C. S. Jongen, J. McCalman and R. G. Bainbridge, "The implementation and evaluation of health promotion services and programs to improve cultural competency: a systematic scoping review," Frontiers in public health, vol. 5, no. 24, 2017. [En línea]. Disponible en https://doi.org/10.3389/fpubh.2017.00024.

[20] L. V. Izaguirre, F. L. Cossio, J. F. R. Pérez y A. O. García, "Impacto de las aplicaciones y servicios informáticos desarrollados por la Universidad de las Ciencias Informáticas para el sector de la salud," Revista Cubana de Informática Médica, vol. 12, no. 1, pp. 58-75, 2020. [En línea]. Disponible http://scielo.sld.cu/scielo.php?script=sci_arttext\&pid=S168418592020000100058.

[21] L. F. Giacometti-Rojas, "Innovación tecnológica y desarrollo de ventaja competitiva en la atención a la salud: enfoque conceptual y metodológico," Revista Gerencia y Políticas de Salud, vol. 12, no. 25, pp. 66-82, 2013. [En línea]. Disponible en https://dialnet.unirioja.es/servlet/articulo?codigo $=7284099$.

[22] F. Lucivero and K. R. Jongsma, "A mobile revolution for healthcare? Setting the agenda for bioethics," Journal of Medical Ethics, vol. 44, no. 10, pp. 685-689, 2018. [En línea]. Disponible en http://dx.doi.org/10.1136/medethics-2017-104741.

[23] J. McCalman, C. Jongen and R. Bainbridge, 'Organizational systems' approaches to improving cultural competence in healthcare: a systematic scoping review of the literature," International journal for equity in health, vol. 16, pp. 78, 2017. [En línea]. Disponible en https://doi.org/10.1186/s12939-017-0571-5.

[24] M. Mi and Y. Zhang, "Culturally competent library services and related factors among health sciences librarians: an exploratory study," Journal of the Medical Library Association: JMLA, vol. 105, no. 2, pp. 132, 2017. [En línea]. Disponible en https://doi.org/10.5195/jmla.2017.203

[25] C. Díaz de León-Castañeda, "Salud electrónica (e-Salud): un marco conceptual de implementación en servicios de salud," Gaceta médica de Mexico, vol. 155, no. 2, pp. 176-183, 2019. [En línea]. Disponible enhttps://www.medigraphic.com/cgi$\mathrm{bin} / \mathrm{new} /$ resumenI.cgi?IDARTICULO $=86526$.

[26] S. M. Hejazi, S. Yadegari and N. Hajrahimi, "Role of intellectual capital on creation of innovation capabilities in HIS and computer units," Journal of Education and Health Promotion, vol. 7, no. 1, 2018. [En línea]. Disponible https://www.ncbi.nlm.nih.gov/pmc/articles/PMC6009149/.

[27] S. H. Yu and M. Y. Chen, "Performance impacts of interorganizational cooperation: a transaction cost perspective," The Service Industries Journal, vol. 33, no. 13-14, pp. 1223-1241, 2013. [En línea]. Disponible en https://doi.org/10.1080/02642069.2013.815729.

[28] N. E. Cho, W. Ke, B. Atems and J. Chang, "How does electronic health information exchange affect hospital performance efficiency? The effects of breadth and depth of information sharing," Journal of Healthcare Management, vol. 63, no. 3, pp. 212-228, 2018. [En línea]. Disponible en doi: 10.1097/JHM-D-16-00041.

[29] C. Akkan, M. A. Karadayi, Y. Ekinci, F. Ülengin, N. Uray and E. Karaosmanoğlu, "Efficiency analysis of emergency departments in metropolitan areas," Socio-Economic Planning Sciences, 69, 100679. New York, USA: Elsevier Science Inc., 2020. [En línea]. Disponible en https://doi.org/10.1016/j.seps.2019.01.001.

[30] D. M. Walker, "Does participation in health information exchange improve hospital efficiency?" Health care management science, vol. 21, no. 3, pp. 426-438, 2018. [En línea]. Disponible en https://link.springer.com/article/10.1007/s10729-017-9396-4. 
[31] R. Hernández-Sampieri and C. Mendoza, Metodología de la investigación. Las rutas cuantitativa, cualitativa y mixta. México: Editorial McGraw Hill, 2018.

[32] S. Aguilar-Barojas, "Fórmulas para el cálculo de la muestra en investigaciones de salud," Salud en Tabasco, vol. 11, no. 1-2, pp. 333338, 2018. [En línea]. Disponible en https://www.redalyc.org/pdf/487/48711206.pdf.

[33] D. Outomuro and A. M. Actis, "Estimación del tiempo de consulta ambulatoria en clínica médica," Revista médica de Chile, vol. 141, no. 3, pp. 361-366, 2013. [En línea]. Disponible en https://scielo.conicyt.cl/scielo.php?pid=S0034$98872013000300012 \&$ script $=$ sci_arttext\&tlng=n

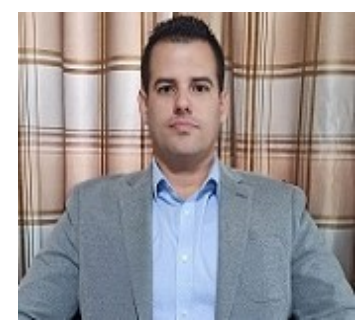

José Felipe Ramírez Pérez. Es Ingeniero en Ciencias Informáticas (2012), Investigador Agregado y Profesor Auxiliar por la Universidad de las Ciencias Informáticas (UCI) de La Habana, Cuba. Se desempeñó como líder científico del Grupo de Investigación de Informática en Salud, como jefe del Departamento de Desarrollo de Aplicaciones y como jefe de Laboratorio de Imágenes Médicas del Centro de Informática Médica de la UCI en el periodo comprendido de octubre de 2014 a enero de 2020. Pertenece a la Sociedad Cubana de Informática Médica y a la Unión de Informáticos de Cuba. Tiene 11 años de experiencia en el análisis, desarrollo y gestión de equipos de desarrollo de software en el área de la Informática Médica, así como en la investigación en las áreas de informática médica, ciencias informáticas y competitividad. Actualmente radica en la Universidad Autónoma de Baja California.

ORCID: https://orcid.org/0000-0002-0765-0685

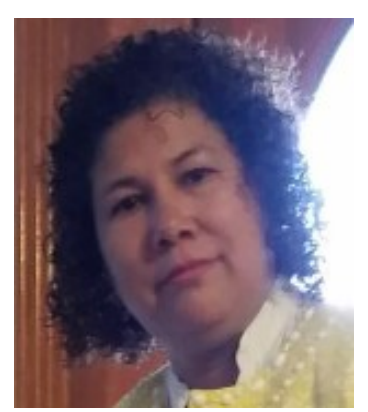

Virginia Guadalupe López Torres. Es Doctora en Ciencias Administrativas (2009) por la Universidad Autónoma de Baja California (UABC), Maestra en Ciencias en Administración Industrial (2001) e Ingeniera Industrial y de Sistemas (1993). Se desempeña como Profesora-Investigadora de la Facultad de Ciencias Administrativas y Sociales de la UABC campus Ensenada. Ha dirigido tesis de posgrado y supervisado Ayudantías de Investigación en licenciaturas y posgrados. Ha dirigido varios proyectos de investigación. Su línea de investigación son los Estudios para el impulso de la competitividad. Cuenta con diversas publicaciones nacionales e internacionales en dichas temáticas. Es miembro del Cuerpo Académico Consolidado "Planeación y Desarrollo: UABC-CA100. Actualmente cuenta con el Perfil Deseable del PRODEP, académico certificado por la Asociación Nacional de Facultades y Escuelas de Contaduría y Administración (ANFECA). Cuenta con la distinción del Sistema Nacional de Investigadores (SNI). ORCID: https://orcid.org/00000002-2795-8951

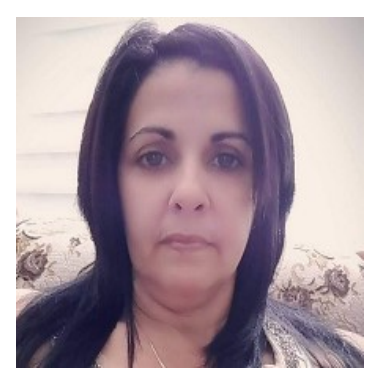

Alicia del Rosario Ramírez Pérez. Es Doctora en Medicina (1998), Especialista de 1 er y 2 do Grado en Medicina General Integral (2003-2009), Especialista de 1er y 2do Grado en Farmacología (2017-2020) y Máster en Longevidad Satisfactoria (2007) por la Universidad de Ciencias Médicas de Villa Clara, Cuba. Es Profesora e Investigadora Auxiliar. Pertenece a la Sociedad Cubana de Farmacología, siendo la presidenta de su Capítulo en Cienfuegos. Investiga en la línea de Envejecimiento poblacional con estudios de utilización de medicamentos en ancianos polimedicados y multimórbidos sobre prevalencia de factores que interfieren en el consumo y evaluaciones de las prescripciones en los niveles de atención primaria y secundaria, diseño de herramientas para deprescripción de medicamentos que conforman planes de tratamiento a largo plazo y sobre herramientas didácticas para la enseñanza de la Farmacología que propicien el uso racional de los medicamentos. Labora en la Universidad de Ciencias Médica de Cienfuegos, Cuba.
ORCID: https://orcid.org/0000-0002-8805-5772.

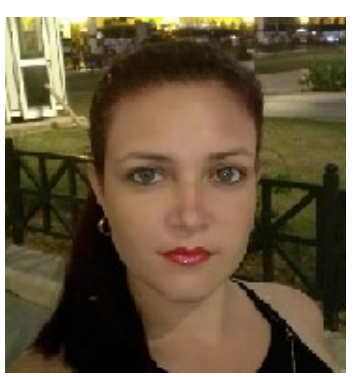

Maylevis Morejón Valdés. Ingeniera en Ciencias Informáticas (2012) por la Universidad de las Ciencias Informáticas, de La Habana, Cuba. Tiene 9 años de experiencia en el desarrollo de softwares de gestión. Se desempeñó como líder de proyectos informáticos en la Universidad de las Ciencias Informáticas. Formó parte de equipos de desarrollo de softwares para la Aduana General de la República de Cuba y para el sector de salud. Pertenece a la Sociedad Cubana de Informática Médica y a la Unión de Informáticos de Cuba. Investiga y trabaja en las áreas de gestión de proyectos informáticos, informática médica, tecnologías de la información y administración. Actualmente radica en la Universidad Autónoma de Baja California, en México, donde estudia la Maestría en Administración de la Facultad de Ciencias Administrativas y Sociales, en el campus Valle Dorado en Ensenada. ORCID:https://orcid.org/0000-0002-5831-9159. 